\title{
Psychosocial Profile and Psychiatric Comorbidity in Patients with Chronic Headache
}

\author{
Jyostna B. ${ }^{1}$, Niveditha Vasireddy ${ }^{2}$ \\ 1,2 Department of Psychiatry, Andhra Medical College, Vishakapatnam, Andhra Pradesh, India
}

\section{ABSTRACT}

\section{BACKGROUND}

Chronic daily headache is a common problem reported in tertiary centres and headache is more commonly comorbid with psychiatric disorders. Headache associated with psychopathology has exaggerated effects on quality of life and disability. Comorbid psychiatric illnesses are risk factors for headaches becoming chronic. Earlier studies on the comorbidity of headache and psychiatric disorders found that comorbidity was found among $90 \%$ of the samples with chronic headaches. The objectives of the study were to evaluate psychiatric comorbidity of patients with chronic headache and the association between socio-demographic factors and psychiatric disorders in patients with chronic headache.

\section{METHODS}

The present study was done in tertiary care hospital, on psychosocial profile and psychiatric disorders in patients with chronic headaches. The subjects belonging to both sexes and above twenty years were included in the study. After obtaining the socio-demographic details of each subject as per pre-decided intake proforma, each subject was administered with M.I.N.I. Plus. The data obtained were analyzed by statistical methods and the results were compiled.

\section{RESULTS}

According to the study, young age group, females, married, illiterates, and people with low socioeconomic status were suffering from chronic headaches. The most common psychiatric comorbidity seen among the study population was major depressive disorder (MDD), dysthymia, panic disorder with agoraphobia and generalized anxiety disorder (GAD).

\section{CONCLUSIONS}

There is a need to assess psychiatric comorbidity among patients suffering from headaches as early diagnosis will help in the good prognosis of the individuals and better patient care.

\section{KEY WORDS}

Chronic Headache, Psychiatric Comorbidity, Major Depressive Disorder, Panic Disorder, Generalized Anxiety Disorder.
Corresponding Author:

Dr. Jyostna B.,

Flat No. 501, China Waltair,

Vishakapatnam, Andhra Pradesh, India.

E-mail: drjyotsnabhukya@gmail.com

DOI: $10.14260 /$ jemds/2022/29

How to Cite This Article:

Jyostna B, Vasireddy N. Psychosocial profile and psychiatric comorbidity in patients with chronic headache. J Evolution Med Dent Sci 2022;11(01):151-156, DOI: 10.14260/jemds/2022/29

Submission 24-12-2021,

Peer Review 30-12-2021,

Acceptance 24-01-2022,

Published 29-01-2022.

Copyright (C) 2022 Jyostna B. et al. This is an open access article distributed under Creative Commons Attribution License [Attribution 4.0 International (CC BY 4.0)] 


\section{BACKGROUND}

Headache is a common human affliction that most often leads people to seek medical advice. Headache has a lifetime prevalence of over $90 \%$ among medical disorders. $10 \%$ of all people report that headache leads to impairment in their daily lives. ${ }^{1}$

Over the life span, $18 \%$ of women and $6 \%$ of men suffer from headaches. Pain-related conditions such as headaches could inflict an enormous burden on the individual, which ultimately translates into direct and indirect costs to society. ${ }^{2}$

Comorbidity is used to indicate a medical condition in a patient that causes, is caused by, or is otherwise related to another condition in the same patient. Headache is commonly comorbid with psychiatric disorders and there is a bimodal relationship with headache and psychiatric disorders. Headache associated with psychopathology has exaggerated effects on quality of life and disability.3,4,5 Comorbid psychiatric illnesses are risk factors for headaches becoming chronic. 6

Chronic, severe headaches affect general functioning and well-being both as a direct and indirect consequence of the attacks. As they are associated with psychiatric syndromes, psychiatrists are often consulted for the evaluation and treatment of people suffering from them. ${ }^{7}$

Earlier studies are there regarding headache and comorbidity but there are few or limited studies regarding headache and psychiatric comorbidity and its relation with psychosocial factors among the individuals.

\section{Objectives of the Study}

- To study psychiatric comorbidity of patients with chronic headache.

- To study the association between socio-demographic factors and psychiatric disorders in patients with chronic headache.

\section{METHODS}

This observational and exploratory study was conducted in a tertiary care hospital between October 2015 and September 2017.

\section{Sample}

A purposive sample of about one hundred patients was taken using a screening questionnaire for chronic headache, after applying the inclusion and exclusion criteria.

\section{Inclusion Criteria}

Patients coming to the outpatient psychiatry department aged 20 years and above.

Patients with chronic headaches defined as headaches occurring daily, more than 15 days per month, for more than 3 months. (As per International Classification of Headache)

\section{Exclusion Criteria}

Patients with organic diseases

Patients not willing to participate in the study.

\section{Operational Procedures}

The study included patients with headaches attending the outpatient department in a tertiary care hospital. Ethical clearance was taken from the Ethics committee of the Institution. Participants were explained about the study and informed consent was taken prior to including them in the study. A detailed history focussed on headache, its nature and course and psychiatric history were taken. A thorough clinical examination with specific reference to blood pressure, pallor, sinus tenderness, refractory error, and neck movements was carried out in all participants as per intake proforma. The Mini Plus was then administered, and the results were tabulated in an excel sheet.

\section{Instruments Used \\ 1. Intake Proforma Which Included \\ a. Socio-demographic data \\ b. Duration of illness \\ c. Past illnesses \\ -Medical \\ -Psychiatric \\ d. Physical examination:}

\section{Mini-International Neuropsychiatric Interview (M.I.N.I. Plus), English Version 5.0.0}

Mini Plus is a short structured diagnostic interview scale, developed jointly by psychiatrists and clinicians from DSMIII/IV and ICD10 based psychiatric disorders. M.I.N.I. Plus was designed for clinical practice and research in a psychiatric primary care setting and takes less time to administer when compared to other similar structured interviews. It includes Alcoholic Dependence / Abuse, Substance Dependence, Psychotic Disorders, Major Depressive Episode, Manic Episode / Bipolar Disorder, Dysthymia, Generalized Anxiety Disorder, Panic Disorder, Agoraphobia, Social Anxiety Disorder, Suicidality, ObsessiveCompulsive Disorder, Posttraumatic Stress Disorder, Anorexia Nervosa, Bulimia Nervosa, Antisocial Personality Disorder.

\section{Statistical Data Analysis}

- The data was analysed using Epi info.

- Descriptive statistics were performed and results were recorded as frequencies.

- $\quad$ The chi-square test was used to estimate P values.

- P-value of $<0.05$ was considered to be statistically significant. 
RESULTS

Table 1 shows the frequency distribution of age, sex, religion, education, occupation, socioeconomic status (SES), marital status and psychiatric disorders. $57 \%$ were diagnosed with major depressive disorder and $10 \%$ with MDD + Melancholia, $28 \%$ with dysthymia, $62 \%$ with suicidality, 22 $\%$ with panic disorder, $7 \%$ with OCD, and $72 \%$ with GAD. ASPD $5 \%$ and alcohol use disorder was seen in $6 \%$ of the subjects.

Among the samples, $35 \%$ of individuals from the young age group and $22 \%$ of individuals from the middle age group were diagnosed with MDD.

Among the subjects diagnosed with MDD (57\%), $28 \%$ were males, $29 \%$ were females, $6 \%$ of individuals were Muslims, $32 \%$ were Hindus and $7 \%$ were Christians. Out of the total sample with MDD, $41 \%$ of individuals were literates and $17 \%$ individuals were illiterates.

In the sample with MDD, $25 \%$ of individuals were unemployed, $30 \%$ of individuals were employed and retired individuals constituted $2 \%$.

Out of the total sample, $29 \%$ of individuals from low SES, $22 \%$ of individuals from middle SES and $6 \%$ of individuals from high SES suffered from MDD respectively.

Among the individuals with MDD, $9 \%$ of individuals were unmarried, $46 \%$ were married and $2 \%$ of individuals were widowed/separated.

Among those with melancholia, the middle age group constituted $9 \%$ and the young age group constituted $1 \%$ of the individuals.

Among the subjects diagnosed with MDD+ mel, $1 \%$ was males, $9 \%$ were females, $6 \%$ were muslims and $4 \%$ of individuals were hindus.

Out of the total sample with MDD+ mel, $5 \%$ of individuals were literates and $5 \%$ were illiterates.

In the sample with MDD+ mel, $6 \%$ of individuals were unemployed and $4 \%$ of individuals were employed.

Out of the total sample, $10 \%$ of individuals from low SES suffered from MDD+ mel.

Among the individuals with MDD+ mel, $8 \%$ were married and $2 \%$ were widowed/separated.

Among subjects diagnosed with dysthymia, young age constituted $28 \%$, males were $9 \%$, females constituted $19 \%$, hindus were $17 \%$, muslims constituted $7 \%$ and $4 \%$ were christians.

The education level of these individuals is, $5 \%$ were illiterates and $23 \%$ were literates. In the sample, $15 \%$ of employed individuals were diagnosed with dysthymia.

In the sample diagnosed with suicidality, $38 \%$ belonged to the young age group (36\% were at low risk, $2 \%$ were at moderate risk) and $24 \%$ belonged to the middle age group (18\% at low risk, $6 \%$ were at moderate risk).

$16 \%$ males were at low risk, $2 \%$ were at moderate risk, $44 \%$ were females of which $38 \%$ were at low risk and $6 \%$ at moderate risk.

Suicidality was seen in $23 \%$ of muslims at low risk, $1 \%$ at moderate risk, $31 \%$ of hindus $(26 \%$ at low risk, $5 \%$ at moderate risk) $5 \%, 2 \%$ at low risk and moderate risk in Christians respectively.

In subjects who were literates, $39 \%$ were at low risk and $3 \%$ at moderate risk.
Among illiterate subjects, $15 \%$ were at low risk and $5 \%$ at moderate risk.

In the unemployed group, $22 \%$ were at low risk, $4 \%$ were at moderate risk, and in the employed group, $28 \%$ were at low risk and $4 \%$ were at moderate risk. Among retired subjects, $4 \%$ were at low risk and suicidality was found.

Suicidality was high in subjects from high SES, $5 \%$ at lowrisk level, $1 \%$ at moderate risk. $11 \%$ in the middle class were at low risk and $45 \%$ among the low class (38\% at lowrisk level and $7 \%$ at moderate risk level). It was also high in unmarried subjects (10\% low, $1 \%$ moderate level), in married individuals, it was $47 \%(42 \%$ low risk, $5 \%$ moderate risk). In widowed subjects, $2 \%$ were at low risk and $2 \%$ at moderate risk.

Among subjects diagnosed with panic disorder, $9 \%$ of them belonged to young age group and $4 \%$ belonged to the middle age group.

Among individuals with panic disorder, males constituted $12 \%$ and females constituted $1 \%$. Panic disorder was seen in $5 \%$ of muslims and $8 \%$ of hindus.

$1 \%$ of illiterates and $12 \%$ literates suffered from panic disorder. It was found among $8 \%$ of employed, $3 \%$ unemployed and $2 \%$ of retired individuals.

Panic disorder in subjects from high SES was $4 \%$ and low SES was $9 \%$.

$2 \%$ of the young age group were diagnosed with panic disorder with agoraphobia and $7 \%$ belonged to the middle age group.

Among individuals with panic disorder with agoraphobia, males constituted $9 \%$ and no female was found with panic disorder with agoraphobia.

Panic disorder with agoraphobia was seen in $1 \%$ of muslims and $8 \%$ of hindus.

$4 \%$ of illiterates and $5 \%$ literates suffered from panic disorder with agoraphobia.

Panic disorder with agoraphobia was found among $3 \%$ of employed, $5 \%$ unemployed and $1 \%$ of retired individuals.

Panic disorder with agoraphobia in subjects from high SES was $2 \%$ and low SES constituted $7 \%$.

In the subjects diagnosed with OCD, $5 \%$ belonged to young age group, $2 \%$ middle age group, $7 \%$ were males, $1 \%$ of muslims, $6 \%$ of hindus, $2 \%$ were illiterates and $5 \%$ were literates.

In unemployed individuals diagnosed with OCD, $3 \%$ were unemployed, $4 \%$ are employed, $5 \%$ of subjects were from middle SES and $2 \%$ from low SES, $5 \%$ were married and $2 \%$ were unmarried.

Among those diagnosed with alcohol use disorder, the majority were males and young age individuals were $5 \%$. Hindus were $6 \%$ and $4 \%$ were literates and most of them belonged to middle socioeconomic status.

Among the total individuals, $48 \%$ of subjects with GAD were from the young age group and $24 \%$ were from the middle age group.

GAD was found among $25 \%$ males and $47 \%$ females. Among them $23 \%$ were muslims, $42 \%$ hindus and $7 \%$ were christians.

Among the individuals with ASPD, $5 \%$ of individuals were young, males respectively. $4 \%$ were hindus and $3 \%$ were literates and all the individuals belonged to middle socioeconomic status. $3 \%$ were married, $2 \%$ were 
unmarried and $3 \%$ of individuals were unemployed and employed constituted $2 \%$ in number.

The chi-square statistics was significant at 0.05 level.

Psychiatric morbidity had statistical significance with some factors such as age, gender, SES, marital status and occupation.

Among subjects with MDE, there was a statistically significant association with age and gender with a P-value of $0.007,<0.05$.

Subjects with panic disorder with agoraphobia had a statistically significant association with age and gender with a P-value of 0.001 and 0.001 respectively.

Among subjects suffering from MDD and melancholia, there was a significant association with age and marital status with a P-value of $0.00002,0.01$ respectively.

Among the subjects suffering from dysthymia, there was a significant association with age and SES with a P-value of 0.001 and 0.05 respectively.

Among the subjects suffering from suicidality, there was a significant association with age, education and occupation with a P-value of $0.002,0.033$, and 0.003 respectively.
Among the subjects suffering from panic disorder, there was a significant association with gender, education, occupation, SES with a P-value of $0.04,0.03$ and 0.0001 respectively.

Among the subjects suffering from panic disorder and agoraphobia, there was a significant association with SES with a P-value of 0.03

Among the subjects suffering from OCD, there was a significant association with gender and SES with P-value of 0.001 and 0.004 respectively.

Among the subjects suffering from AUD, there was a significant association with gender and SES with P-values of 0.02 and 0.0009 respectively.

Among the subjects suffering from GAD, there was a significant association with gender, occupation and SES with P-values of $0.007,0.04$ and 0.001 respectively.

Among the subjects suffering from ASPD, there was a significant association with gender and SES with P-values 0.0006 and 0.0001 respectively.

\begin{tabular}{|c|c|c|c|c|c|c|c|c|c|c|c|c|c|c|c|c|c|c|c|}
\hline \multirow[b]{2}{*}{$\begin{array}{l}\text { Psychiatric } \\
\text { Disorders }\end{array}$} & \multirow[b]{2}{*}{$\mathbf{n}$} & \multicolumn{2}{|c|}{ Age } & \multicolumn{2}{|c|}{ Sex } & \multicolumn{3}{|c|}{ Religion } & \multicolumn{2}{|c|}{ Education } & \multicolumn{3}{|c|}{ Occupation } & \multicolumn{3}{|c|}{ SES } & \multicolumn{3}{|c|}{ Marital Status } \\
\hline & & 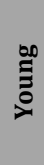 & $\frac{\stackrel{0}{\frac{0}{0}}}{\frac{0}{\Sigma}}$ & $\frac{0}{\Sigma}$ & 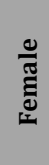 & $\stackrel{\Xi}{\Xi}$ & 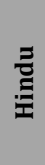 & 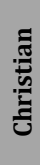 & 冚 & 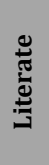 & 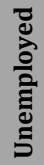 & 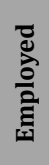 & 总 & 㐫 & $\begin{array}{l}\frac{0}{0} \\
\frac{0}{2}\end{array}$ & 获 & 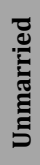 & 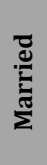 & 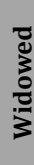 \\
\hline MDE & 57 & 35 & 22 & 28 & 29 & 18 & 32 & 7 & 16 & 41 & 25 & 30 & 2 & 29 & 22 & 6 & 9 & 46 & 2 \\
\hline MD+Melancholia & 10 & 1 & 9 & 1 & 9 & 6 & 4 & 0 & 5 & 5 & 6 & 4 & 0 & 10 & 0 & 0 & 0 & 8 & 2 \\
\hline Dysthymia & 28 & 28 & 0 & 9 & 19 & 7 & 17 & 4 & 5 & 23 & 13 & 15 & 0 & 18 & 10 & 0 & 5 & 23 & 0 \\
\hline Suicide & 62 & 38 & 14 & 18 & 44 & 24 & 31 & 7 & 20 & 42 & 26 & 32 & 4 & 45 & 11 & 6 & 11 & 47 & 4 \\
\hline Panic Disorder & 13 & 9 & 4 & 12 & 1 & 5 & 8 & 0 & 1 & 12 & 3 & 8 & 2 & 9 & 0 & 4 & 2 & 11 & 0 \\
\hline PD with Agoraphobia & 9 & 2 & 7 & 9 & 0 & 1 & 8 & 0 & 4 & 5 & 5 & 3 & 1 & 7 & 0 & 2 & 1 & 8 & 0 \\
\hline OCD & 7 & 5 & 2 & 7 & 0 & 1 & 6 & 0 & 2 & 5 & 3 & 4 & 0 & 2 & 5 & 0 & 2 & 5 & 0 \\
\hline Alcohol use disorders & 6 & 5 & 1 & 4 & 2 & 0 & 6 & 0 & 2 & 4 & 3 & 3 & 0 & 1 & 5 & 0 & 1 & 5 & 0 \\
\hline GAD & 72 & 48 & 24 & 25 & 47 & 23 & 42 & 7 & 23 & 49 & 37 & 33 & 2 & 50 & 22 & 0 & 12 & 56 & 4 \\
\hline ASPD & 5 & 5 & 0 & 5 & 0 & 1 & 4 & 0 & 2 & 3 & 3 & 2 & 0 & 0 & 5 & 0 & 2 & 3 & 0 \\
\hline
\end{tabular}

\section{DISCUSSION}

Depression and headache have a bidirectional relationship where depression increases the risk for chronic daily headaches, and complications associated with chronic headaches may precipitate depressive episodes.

Depression is commonly comorbid with headaches and antidepressants play a major role in relieving headaches. The action of antidepressants on norepinephrine and the serotonin receptors helps in minimising the pain associated with depression. ${ }^{8}$

Chronic daily headache is a common problem in tertiary centres and headache is more commonly comorbid with psychiatric disorders. Headache associated with psychopathology has exaggerated effects on quality of life and disability. $3,4,5$ Comorbid psychiatric illnesses are risk factors for headaches becoming chronic. ${ }^{6}$

Earlier studies on the comorbidity of headache and psychiatric disorders found that comorbidity was found among $90 \%$ of the samples with chronic headaches. ${ }^{9}$ These results laid the contribution to finding the comorbidity of psychiatric disorders in the present study.
For some patients, psychological issues need to be directly addressed e.g., psychiatric comorbidity, difficulties coping with headache, significant problems with sleep and/or stress, medication overuse, and where there is a history of abuse.

In India, limited studies were done in the past comparing socio-demographic variables and psychiatric morbidity in patients with a chronic headache though there were studies of psychiatric morbidity.

\section{Socio-Demographic and Clinical Profile of Chronic Headache \\ Age}

The study group included patients in the age range between 20 and 60 years.

This indicates that the majority of headache problems occur in the young population aged twenty to forty years, most individuals belong to the young age group. It shows that most chronic headache sufferers are between age twenty and forty years.

In the current study, most of the individuals with headaches had a young age onset. Previous studies by Sharma et al. ${ }^{10}$ on psychiatric comorbidity of headache found that majority of the individuals were between twenty and sixty 
years of age, which is contrary to the findings of the present study.

\section{Gender}

Previous literature on the relationship with gender and headache found that females were more prone to headaches than males and women were 2-3 times as likely as men to suffer from chronic headache. ${ }^{11}$ These findings are similar to the study done by Maurizio Pompili11 that women are four times more likely to develop migraine and two times more likely to develop major depression compared with men.

The current study reveals that females' outnumbered males in the number of cases of chronic headache, but that might also be because a similar percentage of the total outpatient department was females. It indicates that more females are suffering from headaches in the general population and thus are seeking treatment for the same.

\section{Marital status}

Regarding headache and marital life, it was observed that headache was more common among married persons which is similar to the findings of $\mathrm{K}$ Madhavi, ${ }^{7}$ that most of the patients suffering from headache are married individuals. The probable cause for this increase in married individuals may be attributed to the fact that chronic headaches have a considerable associated psychological comorbidity. More number of psychosocial problems is likely to be encountered by married persons as compared to unmarried persons, probably predisposing them to this primary headache disorder. ${ }^{12}$

The current study found that four individuals who were widowed were stated to be suffering from headaches which support the finding of Shah et al. ${ }^{10}$ who determined that single subjects or those who lost a spouse were more susceptible to headache or depression.

\section{Socio Economic Status}

In the current study, majority of individuals constituted low socioeconomic status which reflected the general population profile in this area.

A previous study on the socio-economic status of the individuals suffering from headaches done by $\mathrm{K}$ Madhavi, found that the majority of the individuals belonged to middle socioeconomic status, which is contrary to the findings in the present study. This may be due to the location of this tertiary care centre in a rural area.

\section{Religion}

In the present study, most of the subjects suffering from chronic headaches were hindus. This is in accordance with the general population trend of various religions. This high representation of hindus could be explained on the basis that, the hospital from where the samples were collected were of a city with a predominantly hindu population.

Thus, to summarise, from this study emerges a profile of people with chronic headaches tending to be young married females, housewives, with primary standard education or being illiterate and belonging to a low socioeconomic status.

\section{Substance Abuse}

In literature, a relationship to alcohol overuse, illicit drug use and a chronic daily headache was reported by Mathew et al. ${ }^{13}$ and substance abuse disorder was more comorbid with headache and migraine was associated with substance abuse, nicotine dependence, and illicit drug use ${ }^{11}$ and there was increased risk of alcohol and drug abuse in migraine sufferers. ${ }^{14}$

In the current study, there was a low prevalence of substance use disorder which may be explained by the fact that most of the patients were females with a traditionally low prevalence of substance use.

\section{Psychiatric Comorbidity \\ Mood and Anxiety Disorders}

A study done by Zwart et al. ${ }^{15}$ and Dr Alvin E. Lake III et al. ${ }^{16}$ stated that affective disorders occur with at least three-fold greater frequency among headache sufferers than among the general population, and the prevalence increases in clinical populations, especially with chronic headache.

Depression and generalized anxiety disorder are by far the predominant psychiatric morbidities in this population. Dysthymia was found in a significantly high prevalence in this sample.

A similar study done in a tertiary care hospital by $\mathrm{K}$ Madhavi $^{7}$ found that most of the depressed patients suffering from headaches and headaches co-morbid with GAD, panic disorder, somatoform disorder and OCD p patients who consult for headaches experience severe disability and impact, and up to one-third of them report anxiety and/or depression.

According to the findings of Sharma, ${ }^{10}$ people suffered from MDD, dysthymia, panic disorder agoraphobia and phobia and these findings are similar to the present study.

According to a study done by Juang KD, 17 more women than men with chronic tension-type headaches had depressive disorders. Additionally, more women than men with transformed migraines had anxiety disorders. Half of the participants with chronic daily headaches had depressive disorders and one quarter had panic disorder which is similar to the findings of this study.

\section{Suicidality}

According to Maurizio Pompili, 11 suicide risks may play a central role in affecting the quality of life of patients with chronic headaches. The pain associated with headache is itself a potential independent risk factor for suicide, particularly among those with chronic headaches or multiple sources of coexisting pain.

Suicidality of low tendency was seen in a high percentage of patients, $54 \%$ all of who had met criteria for major depressive disorder and who tended to be from low socioeconomic status supports the finding of Wang SJ et al.18 that the presence of chronic headache was a major predictor for the suicidality.

There are significant age and gender differences in comorbidity. The young age group $71 \%$ and females $72 \%$ are significantly more likely than the male individuals to receive lifetime diagnoses of both chronic HA and major depression by age 30 , with relative female risk increasing for CHA in late adolescence and major depression after about age 20 . Longitudinal data indicate that relative to men, women are 
four-fold more likely to develop CHA and two-fold more likely to develop major depression. ${ }^{17}$

\section{CONCLUSIONS}

The majority of headache problems occur in the young population aged 20 to 40 years. Chronic headache found that females outnumbered males in the number of cases. More psychosocial problems were likely to be encountered by married persons as compared to unmarried persons, probably predisposing them to these primary headache disorders. Headache sufferers were mostly with primary standard education or being illiterate and belonging to a low socioeconomic status. Affective disorders occur with at least three-fold greater frequency among headache sufferers than among the general population. Depression and generalized anxiety disorder (GAD) are by far the predominant psychiatric morbidities in this population. A mixed diagnosis of mood, GAD and suicidality was seen in half the patients. Panic disorder was also over-represented in this population. The young age group and females were significantly more likely than the male individuals to receive lifetime diagnoses of both chronic headache and major depression by age 30 . There is a need to assess psychiatric comorbidity among patients suffering from headaches as early diagnosis will help in the good prognosis of the individuals and better patient care.

Data sharing statement provided by the authors is available with the full text of this article at jemds.com.

Financial or other competing interests: None.

Disclosure forms provided by the authors are available with the full text of this article at jemds.com.

\section{REFERENCES}

[1] Merikangas KR, Merikangas JR, Angst J. Headache syndromes and psychiatric disorders: association and familial transmission. J Psychiatr Res 1993;27(2):197210.

[2] Mengistu G, Alemayehu S. Prevalence and burden of primary headache disorders among a local community in Addis Ababa, Ethiopia. J Headache Pain 2013;14(1):30.

[3] Boye B, Lundin KE, Leganger S, et al. The INSPIRE study: do personality traits predict general quality of life (Short form-36) in distressed patients with ulcerative colitis and Crohn's disease? Scand J Gastroenterol 2008;43(12):1505-13.
[4] Lanteri-Minet M, Radat F, Chautard MH, et al. Anxiety and depression associated with migraine: influence on migraine subjects' disability and quality of life, and acute migraine management. Pain 2005;118(3):319-26.

[5] Beghi E, Allais G, Cortelli P, et al. Headache and anxietydepressive disorder comorbidity: the HADAS study. Neurol Sci 2007;28 Suppl 2:S217-9.

[6] Gambini O, Islam L, Demartini B, et al. Psychiatric issues in patients with headaches. Neurol Sci 2010;31 Suppl 1:S111-3.

[7] Madhavi K, Mohan PK, Pasupula SK. Clinical patterns and psychiatric comorbidity of headache: a study from tertiary hospital. Int J Indian Psychol 2016;3:149-53.

[8] Su JA, Tsai CS, Hung TH, et al. Change in accuracy of recognising psychiatric disorders by non psychiatric physicians: Five year data from a psychiatric consultation-liaison service. Psychiatry Clin Neurosci 2011;65:618-23.

[9] Fasmer OB, Oedegaard KJ. Clinical characteristics of patients with major affective disorders and comorbid migraine. World J Biol Psychiatry 2001;2(3):149-55.

[10] Sharma H, Shah S. Psychiatric comorbidity of headache in a medical relief camp in a rural area. Indian J Psychiatry 2006;48(3):185-8.

[11] Pompili M, Serafini G, Di Cosimo D, et al. Psychiatric comorbidity and suicide risk in patients with chronic migraine. Neuropsychiatr Dis Treat 2010;6:81-91.

[12] Sandrini G, Manzoni GC, Zanferrari C, et al. An epidemiological approach to the nosography of chronic daily headache. Cephalalgia 1993;13 Suppl 12:72-7.

[13] Mathew NT, Stubits E, Nigam MP. Transformation of episodic migraine into daily headache: analysis of factors. Headache 1982;22(2):66-8.

[14] Breslau N, Davis GC, Andreski P. Migraine, psychiatric disorders, and suicide attempts: an epidemiologic study of young adults. Psychiatry Res 1991;37(1):11-23.

[15] Zwart JA, Dyb G, Hagen K, et al. Depression and anxiety disorders associated with headache frequency. The Nord-Trøndelag health study. Eur J Neurol 2003;10(2):147-52.

[16] Lake AE, Rains JC, Penzien DB, et al. Headache and psychiatric comorbidity: Historical context, clinical implications and research releavence. Headache 2005;45(5):493-506.

[17] Juang KD, Wang SJ, Fuh JL, et al. Comorbidity of depressive and anxiety disorders in chronic daily headache and its subtypes. Headache 2000;40(10):81823.

[18] Wang SJ, Juang KD, Fuh JL, et al. Psychiatric comorbidity and suicide risk in adolescents with chronic daily headache. Neurology 2007;68(18):1468-73. 